\title{
Treatment of a central venous perforation caused by dialysis intubation using coils and cyanoacrylate glue: A case report
}

\author{
CHUN-ZE ZHOU ${ }^{1,2}$, KAI-CAI LIU ${ }^{2}$, PENG WANG $^{3}$, WEI REN $^{3}$ and WEI-FU LV LV $^{1,2}$ \\ ${ }^{1}$ Department of Interventional Radiology, Medical College of Shandong University, Jinan, Shandong 250021; \\ Departments of ${ }^{2}$ Interventional Radiology and ${ }^{3}$ Nephrology, The First Affiliated Hospital of USTC, Division of \\ Life Sciences and Medicine, University of Science and Technology of China, Hefei, Anhui 230001, P.R. China
}

Received November 30, 2018; Accepted June 6, 2019

DOI: $10.3892 /$ etm.2019.7923

\begin{abstract}
Central venous catheter is one of the most commonly used vascular therapies in patients receiving hemodialysis and vascular perforation is a rare but serious complication. The present study reports on a case of a 64-year-old female who developed massive hemothorax and hemorrhagic shock after long-term dialysis due to central venous perforation during placement of the central venous catheter. This case was successfully managed by digital subtraction angiography-guided direct injection of coils and cyanoacrylate glue into the sinus tract. In addition, the literature regarding central venous perforation resulting from long-term dialysis catheters was reviewed, risk factors and prevention strategies were summarized and the advantages and disadvantages of various therapeutic approaches were compared.
\end{abstract}

\section{Introduction}

Central venous catheter (CVC) is frequently used for temporary or permanent vascular access for hemodialysis, leading to potential complications, including local injury, hematoma, pneumothorax, thrombosis, vein stenosis and catheter-associated infections (1). Central venous perforation is a rare but serious complication that may lead to massive hemorrhage, hemorrhagic shock (HS) and even death. Treatment methods for CVC perforation have varied in the past decade and have included open surgical repair (2-6) and endovascular stent grafting repair (7-9). The present study reports on a case of left brachiocephalic vein (BCV) perforation into the mediastinum and pleura leading to massive hemothorax and HS that was successfully treated by coil and glue embolization of the sinus tract during digital subtraction angiography (DSA) after effective anti-shock therapy.

Correspondence to: Dr Wei-Fu Lv, Department of Interventional Radiology, The First Affiliated Hospital of USTC, Division of Life Sciences and Medicine, University of Science and Technology of China, 17 Lujiang Road, Luyang, Hefei, Anhui 230001, P.R. China E-mail: weifulv@ustc.edu.cn

Key words: central venous, dialysis catheter, perforation

\section{Case report}

The present study reported a case of left BCV perforation that was diagnosed at the First Affiliated Hospital of the University of Science and Technology of China (USTC; Hefei, China). The present study was approved by the Ethics Committee of The First Affiliated Hospital of the USTC and informed consent was also obtained from the patient. A 64-year-old female with a 15-year history of hypertension underwent left forearm arteriovenous fistula (AVF) surgery to treat stage- $\mathrm{V}$ chronic kidney disease (CKD) five years previously. One month prior to presentation at our department on June 8,2017, the patient had a fistula obstruction and a local hospital placed a temporary catheter in the patient's right femoral vein for hemodialysis. However, the inserted catheter caused occlusion of the right BCV. Review of the patient's medical history revealed that she received a long-term hemodialysis catheter (hemosplit $14.5 \mathrm{~F} / 23 \mathrm{~cm}$; BARD) via the left internal jugular vein (LIJV) two days prior to occurrence of HS. LIJV punctures using the Seldinger technique were performed under local anesthesia, followed by smooth introduction and positioning of the guidewires in the left IJV to a level just below the diaphragm. After dilator and guidewire removal, the catheters were introduced, followed by removal of the peel-away sheaths. When placing the catheters, the surgeon felt a marked resistance. Furthermore, the patient reported mild pain and discomfort in the left chest during the catheterization process. After the completion of catheterization, hard venous blood was aspirated. The surgeon considered the possibility of acute thrombosis. A total of 200,000 units of urokinase were injected into the catheter, revealing obstructed flow after $20 \mathrm{~min}$. Subsequently, the patient developed symptoms of palpitations, chest stuffiness and cold sweats. Thoracic anteroposterior radiography at the emergency department indicated that the body segment of the dialysis catheter was located on the left side of the mediastinum (Fig. 1). Therefore, the patient was rapidly transferred to our hospital. Upon admission, general and systemic examinations were unremarkable except for pectoralgia on the left side and chest tightness accompanied by asthma or breathing difficulties/obstruction, an elevated heart rate (115 beats/min) and hypotension (blood pressure, $82 / 45 \mathrm{mmHg}$ ). Laboratory investigations revealed a red blood cell count of $1.72 \times 10^{12} / 1$ (normal range: $3.5 \sim 5.5 \times 10^{12} / 1$ ) and a hemoglobin level of $59 \mathrm{~g} / \mathrm{l}$ (normal range: 110-150 g/l). A contrast-enhanced 
chest computed tomography scan revealed that the left $\mathrm{BCV}$ was perforated through the mediastinum and entered the left thoracic cavity, with the appearance of pleural effusion (Fig. 2a-d). A pleural puncture was performed to extract nonclotting blood. According to the above symptoms, the patient was diagnosed with central venous perforation and HS. The patient immediately received anti-shock treatment by fluid infusion and blood transfusion. The patient was given $6 \mathrm{U}$ total transfused red blood cells and $800 \mathrm{ml}$ fresh frozen plasma. Subsequently, the patient's blood pressure rose to $90-100 / 50-60 \mathrm{mmHg}$ and the heart rate was reduced to $100-110$ beats/min. However, there were obvious symptoms of pectoralgia, which was aggravated when breathing and coughing. Since the patient had evidence of a bleeding disorder with unstable vital signs, it was decided to perform intracavitary treatment to repair the damaged blood vessels. A total of $10 \mu \mathrm{l}$ non-ionic contrast medium (Iodixanol; $27 \mathrm{~g} / 100 \mathrm{ml}$; Amersham Health) was injected into the dialysis catheter, and X-ray fluoroscopy indicated that the contrast agent entered the left thoracic cavity through the front of the dialysis catheter and quickly dispersed (Fig. 3).

A puncture needle was inserted into the left femoral vein, which was implanted with the $10 \mathrm{~F}$ catheter sheath. Angiography revealed that steady blood flow was present in the left subclavian vein (LSV), cephalic vein and superior vena cava with no obvious leakage of the contrast agent. A guidewire (RFxPA35263M; $260 \mathrm{~cm}$; Terumo Corp.) was left in the LSV to seal the sinus groove in the case of unsuccessful sinus embolization. A 4F catheter (Yashiro; Terumo Corp.) was inserted into the sinus canal through the artery end of the original dialysis catheter, followed by a 3F microcatheter (Progreat; Terumo Corp.). A total of four coils $(6 \times 50 \mathrm{~mm}$, Fibered Platinum Coil; Boston Scientific) were then anchored at the fistula through the catheter. Cyanoacrylates [n-butyl-2-cyanoacrylate (NBCA)/n-octyl cyanoacrylate; Fuaile] were mixed with lipiodol (Guerbet) at a proportion of 1:2 and $1.5 \mathrm{ml}$ was injected under fluoroscopic guidance. The long-term dialysis catheter was then replaced at the original puncture site under fluoroscopy and placed in the superior vena cava. Post-operative blood transfusion, fluid infusion and chest drainage were continued. The HGB level increased to $103 \mathrm{~g} / \mathrm{l}$ on the third day after the operation, and the patient was discharged from the hospital. The patient was followed up with for one year and she did not experience any hemorrhage or central venous stenosis.

\section{Discussion}

According to the clinical practice guidelines of the National Kidney Foundation Kidney Disease Outcomes Quality Initiative, the success rates of autologous and grafted internal AVFs are 80 and $10 \%$, respectively (1). However, for various practical reasons, CVC remains the first access for dialysis therapy for most patients. In China, particularly in underdeveloped areas, a large number of patients use long-term dialysis catheters as the only method of vascular access or while waiting for AVF maturation (10). However, dialysis catheters are reported to result in complications, including local injury, hematoma, pneumothorax, thrombosis, venous stenosis and catheter-associated infection. Central venous perforation is a rare complication with frequently occurring serious consequences that even include death. A total of 13 case reports on central venous perforation



Figure 1. Chest X-ray revealing a dialysis catheter inserted into the left thoracic cavity through the mediastinum (scale bar=10 $\mathrm{cm}$ ).

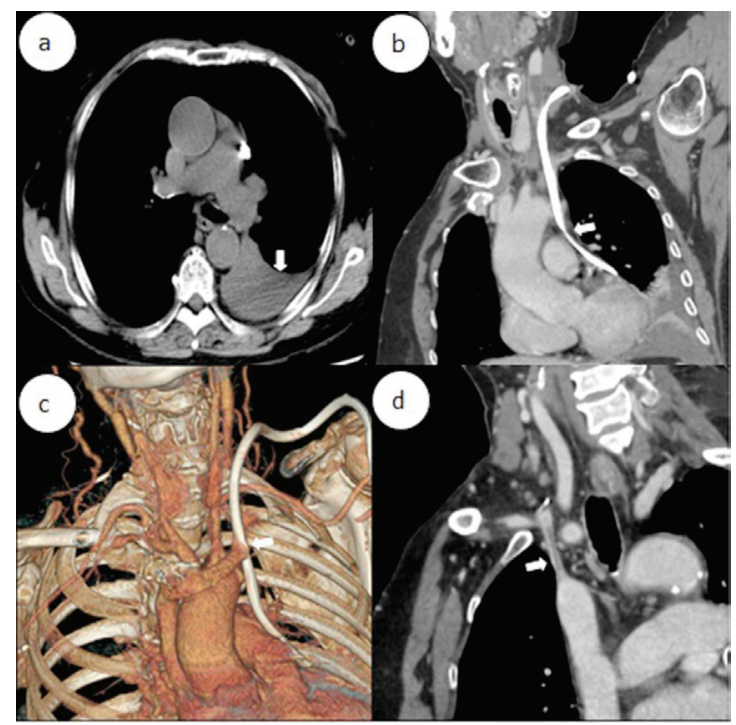

Figure 2. (a) the arrows indicated left pleural effusion. (b and c) the arrows indicated catheter passing through the left brachiocephalic vein into the left thoracic cavity. (d) The arrows indicated right brachiocephalic vein occlusion.



Figure 3. (a) Chest X-ray revealing that the contrast agent entered the left thoracic cavity through the front of the dialysis catheter and dispersed quickly. (b) Left subclavian venography indicating steady blood flow. (c) Radiograph demonstrating catheter in sinus tract after deployment of angiographic coils; (d) Retrograde cholangiogram after application of N-butyl cyanoacrylate glue demonstrating the cessation of the contrast agent leak; (e) angiography demonstrating good sealing of the fistula after removing the dialysis catheter; (f) semipermanent dialysis tube placed in the superior vena cava. 
Table I. Previously published cases of central venous perforation caused by dialysis catheter $(n=13)$.

\begin{tabular}{|c|c|c|c|c|c|c|}
\hline First author (year) & $\begin{array}{l}\text { Puncture } \\
\text { approach }\end{array}$ & Perforation site & Symptoms & Treatment & Outcomes & (Refs.) \\
\hline Winkes (2016) & LIJV & LBCV mediastinum & Pectoralgia hemothorax HS & Thoracotomy & Recovery & (2) \\
\hline Iwańczuk (2013) & RIJV & RBCV pleural cavity & Pectoralgia hemothorax HS & Thoracotomy & Death & (3) \\
\hline Wong (2016) & RIJV & Precava PA LA & Pectoralgia & Thoracotomy & Recovery & (4) \\
\hline Turkyilmaz (2017) & LSV & Precava pleural cavity & Pectoralgia hemothorax HS & Thoracotomy & Recovery & (5) \\
\hline Kuzniec (2010) & RIJV & Precava & NA & $\begin{array}{l}\text { Thoracoscopic } \\
\text { surgery }\end{array}$ & Recovery & (6) \\
\hline Song (2015) & RIJV & Precava & Pectoralgia hemothorax HS & EVSGR & Recovery & $(7)$ \\
\hline Azizzadeh (2007) & LSV & Precava & Hemothorax HS & EVSGR & Recovery & $(8)$ \\
\hline Pua (2014) & LIJV & LBCV & NA & EVSGR & Recovery & (9) \\
\hline Zhang (2016) & RIJV & RBCV mediastinum & Hemothorax & Coils & Recovery & (11) \\
\hline Wetzel (2017) & LIJV & LBCV & Hemothorax HS & $\begin{array}{l}\text { Conservative } \\
\text { therapy }\end{array}$ & Recovery & (12) \\
\hline Kabutey (2013) & LSV & $\mathrm{LBCV}$ & Hemothorax HS & $\begin{array}{l}\text { Conservative } \\
\text { therapy }\end{array}$ & Recovery & (13) \\
\hline Chao (2010) & RIJV & Precava & Pectoralgia HS & $\begin{array}{l}\text { Conservative } \\
\text { therapy }\end{array}$ & Recovery & (14) \\
\hline Garcarek (2015) & LIJV & LBCV mediastinum & NA & Coils and glue & Recovery & $(15)$ \\
\hline
\end{tabular}

EVSGR, endovascular stent grafting repair; HS, hemorrhagic shock; LA, left atrium; L/RBCV, left/right brachiocephalic vein; L/RIJV, left/right internal jugular vein; LSV, left subclavian vein; PA, pulmonary artery; NA, not available.

caused by dialysis catheterization published between 2007 and 2018 were reviewed (Table I) (2-9,11-15). Among them, 8 patients presented with hemothorax and HS (2,3,5,7,8,12-14), and 1 patient died despite rescue after surgery (3).

Review of the relevant literature and summary of our experience led to the assumption that the following factors may increase the risk of central venous perforation caused by dialysis catheters. First, since the distance from the LIJV to the right atrium is longer and passes through the left BCV and the superior vena cava, central venous perforation occurs more frequently with left intubation (2). Second, obesity is a risk factor for central venous perforation (16). Wicky et al (17) retrospectively analyzed 11 cases of severe vascular complications after CVC misplacement, and 7 patients were obese with a body mass index of $>30 \mathrm{~kg} / \mathrm{m}^{2}$. Third, the experience of the surgeons is an important factor affecting central venous perforation (2). The procedure was performed by a primary-hospital kidney physician who had placed $<50$ long-term catheter implants.

To reduce the occurrence of central venous perforation, the following suggestions should be considered. First, the 'Fistula First Initiative' principle should be followed, which may reduce CVC placement, thrombosis and stenosis. The 'Fistula First Initiative' emphasizes the primacy of the AVF as the desired vascular access for patients maintained on chronic hemodialysis (18). Second, the left approach should be considered unless there is a contraindication regarding right intubation. Third, if possible, placement of the CVC should be performed under $\mathrm{X}$-ray guidance. Prior to implantation, vascular conditions may be clearly confirmed through angiography and the position of the catheter may be dynamically viewed under intra-operative fluoroscopy (1). Finally, if the guide wire inside the long-term dialysis catheter is more flexible and the expansion tube is more rigid, even if the guide wire enters smoothly, the rigid expansion tube may break the guide wire. Therefore, the use of more supportive guide wires during the left intubation may be a safer choice. Following placement of the avulsion sheath, the rigid guide wire may be retained in the upper vena cava and the dialysis catheter may be placed in the appropriate position through this guide wire. If the catheter is difficult to maneuver around the turns of blood vessels, the rigid guide wire may be inserted into the two catheter cavities to increase the supporting force.

After central venous perforation, the procedure for dealing with angiorrhexis to prevent further bleeding is a problem that requires to be considered. As presented in Table I, among the 13 cases reported from 2007 to 2018, 4 cases underwent thoracotomy (2-5), 1 case underwent thoracoscopic surgery (6), 3 cases received covered stents (7-9), 1 case received coil embolization (11), 3 cases received conservative treatment (12-14) and 1 case received coil combined with gel embolization (15). Of all of the cases, one patient died after thoracotomy (3). In the present case, therapeutic strategies were required to address the following two important issues. First, due to hemothorax and HS, extubation and hemostasis were the primary problems. Second, due to the patient's right BCV occlusion and poor peripheral vascular conditions, the LIJV channel required to be retained as much as possible. Catheter insertion from the left side was avoided, as there was a contraindication of right intubation, and it was attempted to reduce the risk of 
complications. Due to the marked surgical trauma, as well as the patient's complex disease history and unstable vital signs, surgical treatment was not the preferred treatment. Although covered stent implantation is an effective method for the treatment of vascular injury, this method was not applicable to the present case, since the vascular rupture was located on the opposite side of the left jugular vein opening, which would be covered by the stent, causing left jugular vein reflux disorder (19). Central venous perforation caused by intubation usually has a clear sinus tract in which the catheter is located, providing a basis for endovascular occlusion. To the best of our knowledge, the present study is the second to report on a case with endovascular embolization treatment using coils combined with glues. In contrast to the present case, in the previously reported case, the patient had less blood loss and stable vital signs (15). The advantages of coils and cyanoacrylate glue embolization have been noted in various studies. For instance, Yavuz et al (20) indicated that patients with varicose veins had significant improvement in venous clinical severity scores and Aberdeen varicose vein questionnaire scores with no skin pigmentation, hematoma, paresthesia, deep vein thrombosis or pulmonary embolism after application of a novel NBCA glue ablation catheter. A retrospective chart review of the long-term outcomes of patients treated with the combined injection of coils and cyanoacrylate glue for bleeding gastric fundal varices demonstrated that the combination therapy appeared safe and may reduce the risk of cyanoacrylate embolization (21). Balloon-protected embolization has been adopted for the endovascular repair of central vein injuries (22). In the present case, the catheter passed through the mediastinum and pleura into the thoracic cavity. It was opted for gradually retracting the dialysis catheter simultaneously with coil embolization, which not only enhanced the effect of the coil embolization but also blocked the small broken blood vessels. This method may achieve hemostasis with good long-term outcomes.

In summary, vascular perforation is a rare complication of CVC placement. Combined injection of coils and cyanoacrylate glue is a minimally invasive, safe and effective treatment method.

\section{Acknowledgements}

Not applicable.

\section{Funding}

The current study was funded by Natural Science Foundation of Anhui Province of China (grant no. 1808085MH254).

\section{Availability of data and materials}

The datasets used and/or analyzed during the present study are available from the corresponding author on reasonable request.

\section{Authors' contributions}

$\mathrm{CZ}$ and WL designed the study and drafted the manuscript. $\mathrm{CZ}, \mathrm{KL}, \mathrm{PW}, \mathrm{WR}$ and WL contributed information on the case and participated in data analysis. All authors approved the final version of the manuscript.

\section{Ethics approval and consent to participate}

The present study was approved by the Ethics Committee of The First Affiliated Hospital of USTC. The patient provided consent to participate.

\section{Patient consent for publication}

The patient provided written informed consent for publication.

\section{Competing interests}

The authors declare that they have no competing interests.

\section{References}

1. Group VAW: Clinical practice guidelines for vascular access. Am J Kidney Dis 48(Suppl 1): 176-247, 2006.

2. Winkes MB, Loos MJ, Scheltinga MR and Teijink JA: Dialysis catheter placement via the left internal jugular vein: Risk of brachiocephalic vein perforation. J Vasc Access 17: e75-e78, 2016.

3. Iwańczuk W, Guźniczak P and Kasperczak J: Hemothorax as a complication of subclavian vein cannulation with haemodialysis catheter-case report. Anaesthesiol Intensive Ther 45: 89-92, 2013.

4. Wong K, Marks BA, Qureshi A and Stemm JJ: Migration of a central venous catheter in a hemodialysis patient resulted in left atrial perforation and thrombus formation requiring open heart surgery. A A Case Rep 7: 21-23, 2016.

5. Turkyilmaz A, Karapolat S, Kilic M and Tekinbas C: The perforation of the superior vena cava secondary to the left subclavian dialysis catheter. Vasc Endovascular Surg 51: 95-97, 2017.

6. Kuzniec S, Natal SR, Werebe Edc C and Wolosker N: Videothoracoscopic-guided management of a central vein perforation during hemodialysis catheter placement. J Vasc Surg 52: 1354-1356, 2010.

7. Song D, Yun S, Cho S, Goo DE and Kim YJ: Latrogenic innominate vein injury by hemodialysis catheter, successful endovascular repair. J Vasc Access 16: e4-e5, 2015.

8. Azizzadeh A, Pham MT, Estrera AL, Coogan SM and Safi HJ: Endovascular repair of an iatrogenic superior vena caval injury: A case report. J Vasc Surg 46: 569-571, 2007.

9. Pua U: Central vein perforation during tunneled dialysis catheter insertion: Principles of acute management. Hemodial Int 18: 838-841, 2014.

10. Wang K, Wang P, Liang X, Lu X and Liu Z: Epidemiology of haemodialysis catheter complications: A survey of 865 dialysis patients from 14 haemodialysis centres in Henan province in China. BMJ Open 5: e007136, 2015.

11. Zhang W, Liu T, Wang X, Huo Y, Jia Y, Su L and Liu Y: Hemothorax caused by replacement of hemodialysis catheter: A case report. Hemodial Int 20: E7-E10, 2016.

12. Wetzel LR, Patel PR and Pesa NL: Central venous catheter placement in the left internal jugular vein complicated by perforation of the left brachiocephalic vein and massive hemothorax: A Case Report. A A Case Rep 9: 16-19, 2017.

13. Kabutey NK, Rastogi N and Kim D: Conservative management of iatrogenic superior vena cava (SVC) perforation after attempted dialysis catheter placement: Case report and literature review. Clin Imaging 37: 1138-1141, 2013.

14. Chao CS, Chao CT, Chin HK and Chang YP: Application of positive end-expiratory pressure in a case with large laceration on the superior vena cava. J Anesth 24: 253-255, 2010.

15. Garcarek J, Wątorek E, Kurcz J, Kusztal M, Gołebiowski T, Letachowicz K, Letachowicz W, Weyde W and Klinger M: Endovascular reparation of central vein injury with balloon-protected embolization. Cardiovasc Intervent Radiol 38: 1057-1059, 2015.

16. Mansfield PF, Hohn DC, Fornage BD, Gregurich MA and Ota DM: Complications and failures of subclavian-vein catheterization. N Engl J Med 331: 1735-1738, 1994.

17. Wicky S, Meuwly JY, Doenz F, Uske A, Schnyder P and Denys A: Life-threatening vascular complications after central venous catheter placement. Eur Radiol 12: 901-907, 2002. 
18. Schinstock CA, Albright RC, Williams AW, Dillon JJ, Bergstralh EJ, Jenson BM, McCarthy JT and Nath KA: Outcomes of arteriovenous fistula creation after the fistula first initiative. Clin J Am Soc Nephrol 6: 1996-2002, 2011.

19. Anaya-Ayala JE, Smolock CJ, Colvard BD, Naoum JJ, Bismuth J, Lumsden AB, Davies MG and Peden EK: Efficacy of covered stent placement for central venous occlusive disease in hemodialysis patients. J Vasc Surg 54: 754-759, 2011.

20. Yavuz T, Acar AN, Aydin H and Ekingen E: A retrospective study of a new n-butyl-2-cyanoacrylate glue ablation catheter incorporated with application guiding light for the treatment of venous insufficiency: Twelve-month results. Vascular 26: 547-555, 2018.

21. Bhat YM,WeilertF, Fredrick RT,Kane SD, Shah JN,Hamerski CM and Binmoeller KF: EUS-guided treatment of gastric fundal varices with combined injection of coils and cyanoacrylate glue: a large U.S. experience over 6 years (with video). Gastrointest Endosc 83: 1164-1172, 2016.
22. Vollherbst DF, Otto R, Do TD, von Deimling A, Kauczor HU, Bendszus M, Sommer CM and Möhlenbruch MA: Extra-small dual-lumen micro-balloon catheters can improve endovascular embolization: An experimental in vivo and in vitro study. J Neurointerv Surg 10: 1092-1096, 2018.

This work is licensed under a Creative Commons Attribution-NonCommercial-NoDerivatives 4.0 International (CC BY-NC-ND 4.0) License. 